\title{
CLINICAL PRACTICE Sudden death on an aeroplane
}

\author{
M Tiemensma, Philip Buys, S A Wadee
}

Air travel by adults with pulmonary disease requires preflight medical evaluation and baseline medical examinations to ensure safe travel. ${ }^{1}$ We present a case of sudden death on an aeroplane caused by tension pneumothorax, in a patient with known pulmonary disease.

The body of a 27-year-old woman was referred to the Tygerberg Forensic Pathology Laboratory for a medico-legal autopsy. She had experienced shortness of breath and chest pain shortly after take-off in a commercial aircraft, and died shortly thereafter. The aircraft was forced to return to the departure airport. Despite medical practitioners having been on board, the diagnosis of a tension pneumothorax was not made. The subject had been on repeat treatment for pulmonary tuberculosis, and her medical history included drainage of pneumothoraces and pleural effusions.

A low-dose X-ray (LODOX) Statscan performed at the Tygerberg Forensic Pathology Laboratory demonstrated a leftsided tension pneumothorax, right shift of the mediastinum, depression of the left hemi-diaphragm, and collapse of the left lung, all of which were confirmed at autopsy (Fig. 1). The lungs had numerous bilateral subpleural bullae, with an area of fresh haemorrhage on the upper lobe of the left lung, consistent with a ruptured bulla. Histology of the lungs showed the presence of fibrosis, nonspecific chronic inflammation and acute fresh haemorrhages in a section taken of the suspected ruptured bulla. No signs of active tuberculosis could be demonstrated.

\section{Discussion}

Medical doctors may be involved in the screening (management) of patients at risk for commercial air travel by performing a pre-flight evaluation, or may encounter an inflight emergency while travelling.

Awareness of medical conditions associated with a risk of flying, the complications thereof, and pre-flight evaluation and recommendations are of the utmost importance to ensure safe air travel for at-risk passengers. Patients with relevant conditions should be informed of the risk of flying, as ignorance can lead to disastrous complications; many such passengers may be unaware of the health implications of air travel. $^{2}$

$M$ Tiemensma and S Wadee are affiliated to the Division of Forensic Medicine, Department of Pathology, and the Forensic Pathology Services Division, Faculty of Health Sciences, Stellenbosch University, Tygerberg, Western Cape. Philip Buys is affiliated to the South African Society of Aerospace and Environmental Medicine, Pretoria.

Corresponding author:M Tiemensma (mtiemensma@sun.ac.za)

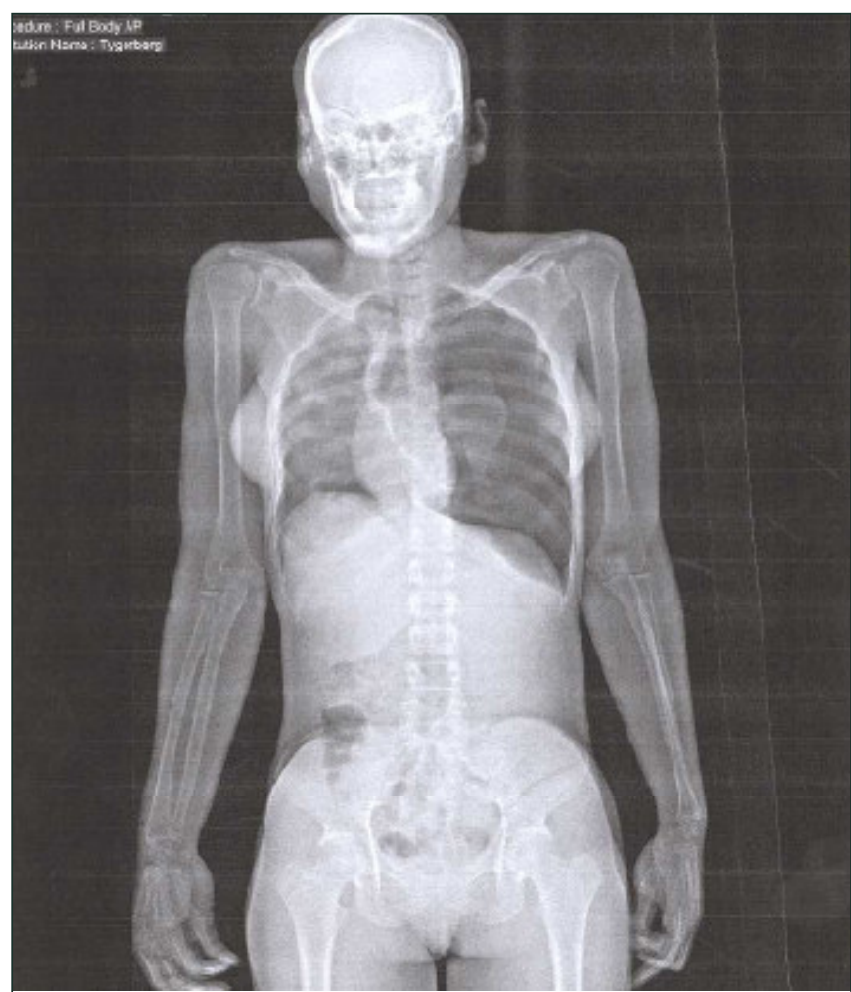

Fig. 1. A LODOX Statscan demonstrates a left-sided tension pneumothorax, right shift of the mediastinum, depression of the left hemidiaphragm, and collapse of the left lung.

Individuals with various pulmonary diseases need special pre-flight evaluation and possible supplemental oxygen delivery during a flight, as the reduction in partial pressure of oxygen $\left(\mathrm{PO}_{2}\right)$ experienced in commercial aviation may exacerbate their symptoms. ${ }^{1}$ Such pulmonary conditions include chronic obstructive airway disease, asthma, restrictive and interstitial lung disease, pulmonary hypertension and

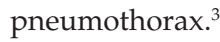

Nowadays, flight tickets can be booked electronically via internet websites. Although information about health conditions is available on the websites, no specific question is asked while booking a flight whether the passenger is healthy and fit to fly - one has to search these sites to find the relevant health- and safety-related information. The onus rests on the passenger with special needs to obtain a medical information sheet from the airline and have it completed by a medical doctor.

The recommendations of the British Thoracic Society Standards of Care Committee ${ }^{4}$ for air travel post-pneumothorax are:

- Follow-up X-rays are to be obtained before air travel to exclude the presence of residual air or recurrence of pneumothorax.

- Patients with a current closed pneumothorax should not travel on commercial flights. 
- Patients may be able to fly 6 weeks after a definitive surgical intervention and resolution of a pneumothorax. Careful medical assessment is required beforehand.

- Patients who have not had surgery must have had a chest radiograph confirming resolution, and at least 6 weeks must have elapsed following resolution before travel.

- Although recurrence is unlikely during a flight, the consequences may be significant, given the absence of prompt medical care. This is particularly true for patients with additional co-existing lung disease. Passengers may wish to consider alternative forms of transport within 1 year of the initial event.

The probability exists that many patients are currently not assessed to determine their fitness for air travel, in consequence of pulmonary, cardiovascular, metabolic and neurological conditions. $^{2}$

We wish to warn attending physicians to inform their patients about medical conditions that may make air travel dangerous; about the importance of pre-flight assessment to ensure safe air travel; and about awareness of possible in-flight emergencies to ensure proper emergency management.

1. Robson AG, Hartung TK, Innes JA. Laboratory assessment of fitness to fly in patients with lung disease: a practical approach. Eur Respir I 2000; 16: 214-219.

2. Silverman D, Gendreau M. Medical issues associated with commercial flights. Lancet 2008; 373 2067-2077

3. Mohr LC. Hypoxia during air travel in adults with pulmonary disease. Am J Med Sci 2008; 335(1): 71-79.

4. British Thoracic Society Standards of Care Committee. Managing passengers with respiratory disease planning air travel: British Thoracic Society recommendations. Thorax 2002; 57(4); 289-304.

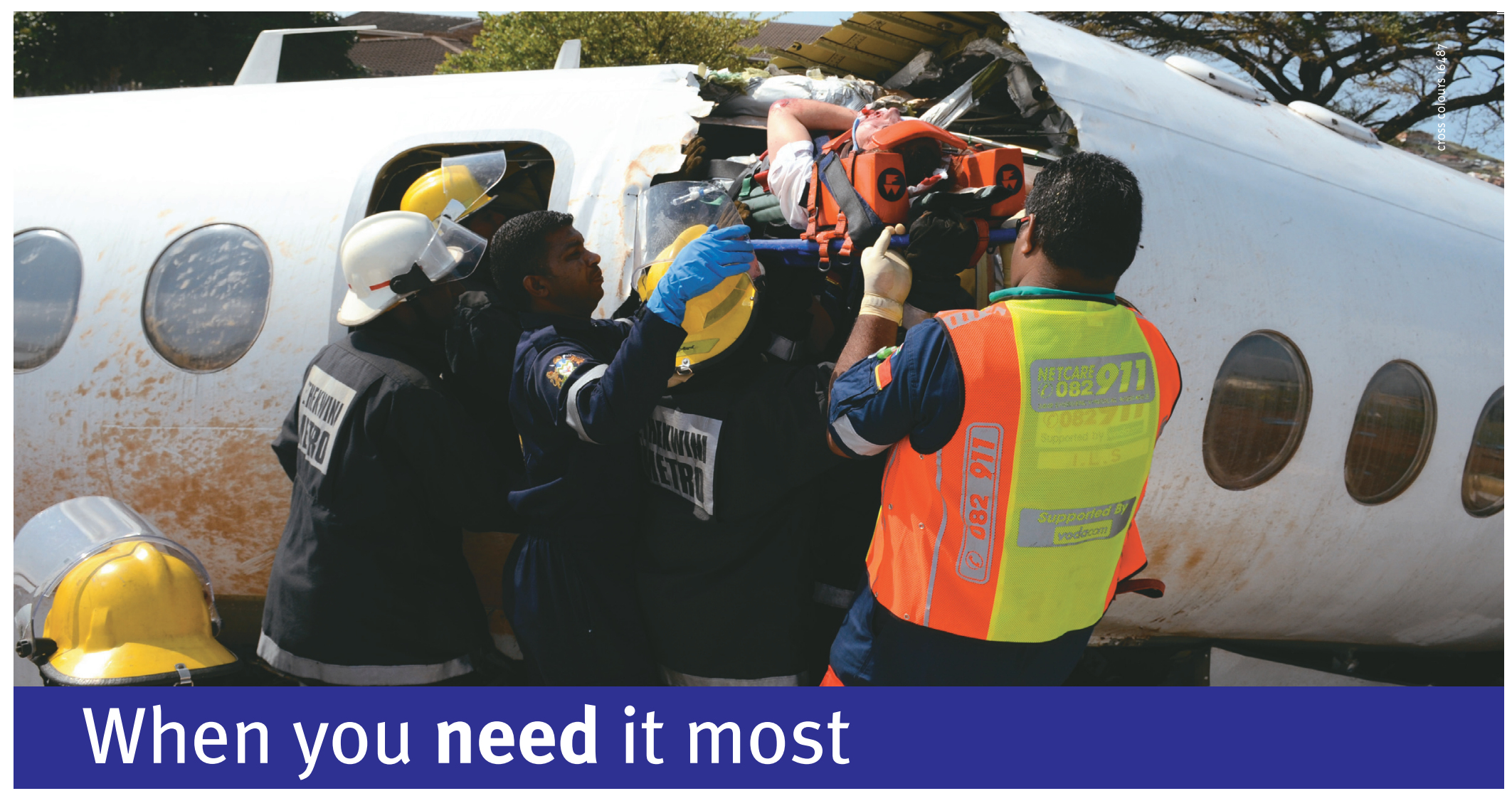

Netcare 911 Emergency Services Team offers you world-class emergency medical assistance, evacuation by road or air and telephonic medical advice. In 2008, South Africa's trust and confidence in Netcare 911 was proved when we were voted the country's top healthcare brand.

Netcare 911. 24 hour emergency medical assistance you can count on. Netcare 911 is an Authorised Financial Services Provider.

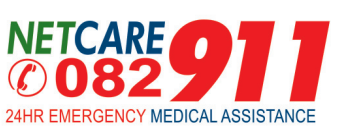

24HR EMERGENCY MEDICAL ASSISTANCE 